\title{
Mirando más allá de los servicios de atención sanitaria: el papel de la salud pública
}

\author{
Blanca Lumbreras \\ Universidad Miguel Hernández, Alicante
}

\section{Resumen}

La salud pública tiene un papel prioritario en la mejora de la salud de la población, a través de los distintos aspectos que se presentan en este artículo y que van más allá de la actuación de los servicios asistenciales. En primer lugar, la salud global, aceptando que los problemas de salud son globales y que requieren acciones a nivel global, así como la necesidad de incorporar la investigación operacional en la toma de decisiones. En segundo lugar, los determinantes sociales y cómo afectan al binomio salud-enfermedad. Asimismo, el desarrollo e implementación de la estrategia Salud en Todas las Políticas y la reducción de la medicalización creciente de la sociedad.

Palabras clave: atención sanitaria, salud pública, salud global, determinantes sociales, medicalización.

Clasificación JEL: I11, I18, I38.

\begin{abstract}
Public health has a priority role in improving the health of the population, through the different aspects presented in this article, that go beyond the performance of health care services. Firstly, global health, accepting that health problems are global and require actions at a global level, together with the need to incorporate operational research into decision-making. Secondly, the social determinants and how they affect the health-disease binomial. Also, the development and implementation of the strategy Health in All Policies, and the reduction of the increasing medicalization of society.
\end{abstract}

Keywords: health care, public health, global health, social determinants, medicalization.

JEL classification: I11, I18, I38.

\section{Introducción}

Los cambios políticos y sociales que se han dado en las últimas décadas a nivel global, la generación de nuevo conocimiento en salud pública en múltiples disciplinas y las innovaciones en la práctica de la salud pública en los ámbitos sanitarios, otorgan un papel relevante a la salud pública en la mejora de la salud poblacional, más allá de la actuación de los servicios asistenciales.

Algunos de los aspectos de la salud pública que pueden incidir con más relevancia en su relación con la atención sanitaria son: la salud global y la gobernanza global en salud; los determinantes sociales y cómo afectan al binomio salud-enfermedad; aspectos relevantes del buen gobierno sanitario, como se describe en el artículo de Ildefonso Hernández en este monográfico; la estrategia Salud en Todas 
las Políticas, y la medicalización creciente de la sociedad, entre otros. Aunque todos estos aspectos están interrelacionados entre sí, para una mayor claridad, en este artículo se expondrán de manera independiente.

Relacionados con todos ellos se encuentran la ética y la salud pública, que han aportado criterios para la consideración de cuestiones que afectan a la salud de la población, tales como es el dispendio en la investigación y el retorno social de la ciencia, la utilización inadecuada de los recursos sanitarios, o la participación del sector privado en acciones de promoción de salud en las que hay obvios conflictos de interés (Hernández-Aguado et al., 2016). La ética, cuyas aplicaciones han estado hasta hace poco menos desarrolladas en el ámbito de la salud pública que en la asistencia sanitaria, está mostrando una gran utilidad en la consideración de las decisiones relativas a las intervenciones en salud pública. En particular, los nuevos marcos de ética en salud pública del siglo XXI aportan las claves para abordar los dilemas éticos planteados por la cuestión sobre quién tiene responsabilidad en la salud de las poblaciones, facilitando la toma de decisiones en aquellas intervenciones que deben armonizar las preferencias individuales y el interés comunitario (Nuffield Council on Bioethics, 2014).

\section{La salud global}

La salud pública y la salud internacional a lo largo del tiempo han realizado esfuerzos en combatir las enfermedades con perspectivas diversas que les han hecho poner el énfasis de su acción bien en problemas específicos de salud, con una mayor preferencia por las enfermedades transmisibles en el caso de la salud internacional, bien en los condicionantes de salud en el caso de la salud pública en los últimos decenios. La salud global, que deviene de ambas, surge a partir de la constatación de que algunos de los condicionantes claves de la salud son supranacionales y por tanto las soluciones deben ser de carácter global. La salud global abarca acciones que van desde la defensa del acceso universal a los servicios de salud a la modificación de la gobernanza global en ámbitos como el comercio, la propiedad intelectual o la cooperación, al objeto de mejorar la salud en términos absolutos a nivel global, así como favorecer la equidad en salud entre los países y entre toda la población en cada país. Uno de los aspectos relevantes de la salud global es que asume la visión más actual de la salud pública, incorporando la visión de los condicionantes sociales de la salud y la necesidad de incluir la salud y la equidad en salud en el conjunto de las políticas públicas y privadas (véase apartado 4) dirigidas a una mejor salud de las poblaciones.

La última revisión de la Carga Global de Enfermedad (Global Burden of Disease), publicada por Lancet, en sus análisis del periodo 1990-2010, muestra cómo los factores de riesgo de enfermedades han cambiado sustancialmente entre los años de estudio, con factores de riesgo que causan enfermedades transmisibles en niños a aquellos relacionados con enfermedades no transmisibles en adultos (Lim et al., 2012). 
Estos cambios se relacionan con el envejecimiento de la población, la disminución de la mortalidad en niños menores de cinco años y cambios en la exposición a factores de riesgo, asociados en muchas ocasiones a la pobreza y a la falta de infraestructuras y recursos sanitarios, así como a la ausencia de políticas públicas saludables en los países en vías de desarrollo, e influyen en las necesidades de salud a nivel global.

En este punto, es importante señalar la importancia de los Objetivos de Desarrollo Sostenible (ODS), también conocidos como Objetivos Mundiales. A finales de septiembre de 2015, después de un largo proceso, la Asamblea General de Naciones Unidas aprobó la Agenda 2030 para el Desarrollo Sostenible que incorpora 17 ODS, estructurados en 169 metas (Resolución aprobada por la Asamblea General de las Naciones Unidas, 25 septiembre de 2015). Estos objetivos se basan en los logros de los Objetivos de Desarrollo del Milenio (ODM), fijados en el año 2000 y que debían cumplirse para el año 2015, aunque incluyen nuevas esferas como el cambio climático, la desigualdad económica, la innovación, el consumo sostenible y la paz y la justicia, entre otras prioridades (Naciones Unidas, 2015). Los ODM tuvieron una vigencia de 15 años, y a pesar de que con su implementación se lograron grandes avances en materia de desarrollo y cuidado del medio ambiente, las desigualdades como problema se dan en prácticamente todos los países, viéndose además un aumento de las mismas. Existen todavía graves problemas de salud y áreas que muestran indicadores básicos sociales y de salud manifiestamente mejorables, así como desigualdades en salud entre países y dentro de los mismos que son injustas.

En este contexto, los ODS retoman aquellos ODM no alcanzados y para los que todavía hay carencias, y además añaden problemas o temáticas que en el año 2000 no fueron planteados. Los ODS entraron en vigor el 1 de enero de 2016, y su importancia radica en su estructura: la interrelación entre todos ellos y, en particular, cómo la mayoría tiene gran impacto en la salud, a la vez que la buena salud contribuye decisivamente al avance de varios de ellos. Asimismo, el marco temporal es también relevante, ya que los países cuentan con 15 años en los que deberán intensificar esfuerzos para poner fin a la pobreza en todas sus condiciones, reducir la desigualdad y realizar acciones contra el cambio climático, con el objetivo de que todos los países los incorporen (Naciones Unidas, 2017).

La salud global concede una gran importancia a los servicios de salud, precisamente porque su universalización forma parte del objetivo 3 de los ODS como meta a alcanzar ( «Garantizar una vida sana y promover el bienestar para todos en todas las edades»). No obstante, señala como esencial introducir mejoras en la calidad del sistema sanitario que estén adaptadas a cada contexto particular y con anterioridad a la instauración de la cobertura sanitaria universal, para que ésta tenga los beneficios esperados. En caso contrario, la universalización podría tener otros efectos en la población como son la aparición de problemas relacionados con la seguridad del paciente, la infrautilización del cuidado basado en la evidencia, la sobreutilización de cuidado no apropiado, la inequidad, etc. (Berwick et al., 2018). Además, y aunque actualmente existe una gran evidencia disponible acerca de las intervenciones sanitarias que son eficaces, apenas se tiene conocimiento acerca de cómo se deben 
implementar dichas intervenciones para que alcancen una elevada eficacia independientemente del entorno, teniendo en cuenta la gran diversidad de sistemas de salud existentes en el mundo. Para alcanzar un adecuado estado de salud en todos los ámbitos, es necesario llevar a cabo investigación operacional, definida como la investigación sobre la implementación de políticas de salud; es decir, identificar intervenciones de eficacia demostrada y aplicarlas en cada contexto concreto (Peters et al., 2014). Esta investigación se debería integrar en todos los procesos de toma de decisiones de políticas de salud y ser colaborativa entre los que forman parte de la formulación de políticas, la gestión de programas de salud y la investigación, así como los destinatarios de la investigación (es lo que se conoce como investigación acción-participativa) (Theobald et al., 2018). Sin embargo, y a pesar de su importancia, este tipo de investigaciones apenas están desarrolladas, tanto por desconocimiento como por falta de financiación. Aspectos como por ejemplo la medicalización, que se discute más adelante en este capítulo, han sido evaluados exclusivamente en el contexto de países desarrollados, por lo que su análisis teniendo en cuenta los distintos entornos sanitarios debería incorporarse también a la agenda de salud global (Clark, 2014). En resumen, sin investigación sobre implementación de políticas, la introducción de intervenciones para mejorar la atención sanitaria puede derivar en un gasto excesivo de recursos que no se vea reflejado en la mejora de la salud de la población a la que van dirigidas.

La salud global reconoce que, además de los servicios de salud, una política sanitaria integral implica a muchos otros ámbitos ajenos a la salud y a muchos otros actores (instituciones privadas, organizaciones no gubernamentales, de la sociedad civil, organizaciones religiosas y académicas, entre otras) que participan activamente en el análisis y diseño de propuestas para responder de forma efectiva y eficiente a las necesidades de salud de la población. Desde distintos ámbitos se han visto movimientos a favor de transformar la salud pública en un movimiento social que apoye las acciones de salud colectiva en todos los niveles de la sociedad a nivel global. De hecho, en los últimos diez años ha habido un crecimiento exponencial de los recursos internacionales dedicados a la promoción de la salud, a través del esfuerzo conjunto de gobiernos, organismos internacionales, ONG y fundaciones filantrópicas. Como ejemplo de estas nuevas alianzas destacan: el Fondo Mundial de Lucha contra el SIDA, la Tuberculosis y la Malaria (GFATM); la Fundación Bill y Melinda Gates; la Alianza Mundial para el Fomento de la Vacunación y la Inmunización (GAVI); la alianza Stop Tuberculosis; la alianza Roll Back Malaria y la Alianza Mundial para Mejorar la Nutrición (GAIN).

No obstante, la proliferación de estas iniciativas también se ha traducido en preponderancias de unos programas de salud sobre otros, en función de los intereses de los donantes y no de las necesidades reales de las poblaciones afectadas, y en un marco organizativo más o menos vertical también en función de las preferencias de algunos donantes. Instituciones como la OMS y otras instituciones multilaterales juegan un papel de liderazgo en la definición de las prioridades en salud, aunque cada vez más, la gobernabilidad de la salud pública se está diluyendo entre estos 
distintos actores implicados. Por un lado, las empresas privadas tienen un rol influyente en la gobernanza global, dado que las grandes empresas trasnacionales tienen un gran poder económico que pueden utilizar para promover sus intereses en la toma de decisiones de las políticas públicas. Las fundaciones, por ejemplo, a través de sus contribuciones a iniciativas de salud global, mejoran la salud de muchos e inspiran a otros a realizar contribuciones económicas similares; sin embargo pueden influir en las políticas públicas marcando su propia agenda, que no siempre coincide con los intereses generales. Además del poder económico, hay otras estructuras con capacidad de influir a través de convicciones acerca de lo que es ético y socialmente aceptable, como son las organizaciones no gubernamentales internacionales, los medios de comunicación o las agrupaciones científicas.

Sobre la gobernanza global de la salud han surgido voces críticas acerca del papel que juega la OMS y la posible presencia de conflictos de interés que pueden influir en sus decisiones y agenda. Una de estas voces es Global Health Watch, que junto con otras organizaciones publica un informe de salud global alternativo (Rowson et al., 2004).

En esta línea crítica, la revista Lancet publicó en el año 2014 un manifiesto sobre la gobernanza global en salud a través de la creación de la Comisión para la Gobernanza Global de la Salud (Ottersen et al., 2014). Esta comisión está motivada por la convicción compartida de que el sistema actual de gobernanza global no protege adecuadamente la salud de la población. Además, este fracaso afecta desigualmente y es especialmente adverso para los más vulnerables, provocando desigualdades en salud.

En resumen, los problemas de salud tienen una dimensión global que está determinada por diversos factores: la aceptación de que los problemas de salud son globales independientemente de la existencia de fronteras y que algunas de sus causas más importantes requieren acciones a nivel global, lo que exige cooperación internacional; el papel de los distintos actores, como son los servicios de salud, ONG, empresas privadas y filántropos, que puede influir en la toma de decisiones públicas y deben considerarse en cualquier análisis sobre la salud global; y el hecho de que la globalización puede crear gradientes de poder e influencia en las políticas causando problemas de salud globales y creando inequidades sociales en salud. Asimismo, para lograr que las intervenciones en salud tengan resultados beneficiosos independientemente del entorno al que se dirigen, es imprescindible incorporar la investigación operacional en la toma de decisiones.

\section{Los determinantes sociales de la salud}

A través del trabajo de investigadores como Sir Michael Marmot, se ha ampliado el conocimiento sobre las causas de los problemas de salud mostrando hasta qué punto diversos condicionantes sociales interrelacionados explican los gradientes de salud en las poblaciones. El marco de los condicionantes sociales de la salud 
propuesto por la Comisión de Determinantes Sociales de la Salud de la OMS dirigida por el profesor Marmot propone que la distribución de la salud y el bienestar son un desenlace (y en ocasiones a su vez causa) derivado de factores a nivel macro (las políticas, el contexto socioeconómico, la gobernanza o las normas y valores de la sociedad que han sido asumidos) que interactúan con los ejes que determinan la posición socioeconómica y con factores a nivel intermedio, junto con el servicio sanitario (circunstancias materiales, cohesión social, conductas, factores biológicos o factores psicosociales) (Marmot, 2008).

Los determinantes sociales de la salud repercuten directamente en esta; permiten predecir la mayor proporción de la varianza del estado de salud (inequidad sanitaria); estructuran los comportamientos relacionados con la salud, e interactúan mutuamente en la generación de salud. En las últimas décadas, las crecientes disparidades de salud en los países desarrollados y entre países en vías de desarrollo y los países desarrollados se han asociado con estos factores sociales. Un análisis de los determinantes sociales de la salud puede ayudar a priorizar las intervenciones más necesarias, cumpliendo uno de los objetivos de la atención sanitaria como es la reducción de las desigualdades sanitarias a nivel regional, nacional e internacional.

La Comisión sobre Determinantes Sociales de la Salud antes mencionada y creada en el año 2008 por la OMS, ha tenido repercusión en todos los niveles, desde la aparición de revistas científicas sobre ámbitos como la salud urbana, a las propias publicaciones de oficinas regionales de la OMS. Esta Comisión reconoce que las desigualdades sociales sesgan la distribución de la salud. Se llega a la conclusión que «las normas sociales, políticas y prácticas que toleran o promueven la injusta distribución y acceso al poder, la riqueza y otros recursos sociales necesarios crean desigualdades sistemáticas en las condiciones de vida». Asimismo, argumenta que las causas fundamentales de la inequidad en salud se centran en la distribución desigual del poder, el dinero y los recursos. Las recomendaciones de la Comisión para actuar sobre los determinantes de la salud son: 1) mejorar las condiciones de vida;2) luchar contra la distribución desigual del poder, el dinero y los recursos; y 3) medir y analizar el problema y evaluar las intervenciones.

Como evaluación de los resultados obtenidos por la Comisión de Determinantes de la Salud, en el año 2013 se publicó el Informe sobre la Salud en Europa 2012, un estudio de las desigualdades en salud entre y dentro de los 53 países miembros de la Región Europea de la OMS, como ayuda al desarrollo del nuevo marco europeo de salud y bienestar denominado Health 2020 (Organización Mundial de la Salud, 2013). Dicho documento evalúa datos entre 2009 y 2010, anteriores a las medidas de recorte en el gasto público sanitario llevado a cabo por diversos países europeos, por lo que no permite ver los efectos que ha tenido la crisis en la salud. En dicho informe se muestra que, aunque el nivel general de salud en toda la Región Europea de la OMS ha mejorado claramente, las estadísticas de salud europeas muestran desigualdades dentro de cada país y entre estos. Uno de los resultados de este informe muestra cómo la esperanza de vida se está incrementando en toda la región, aumentando en cinco años desde 1980 hasta llegar a 76 años en 2010. Sin embargo, 
subsisten grandes desigualdades en la esperanza de vida entre hombres y mujeres, entre países y entre grupos de población.

El Informe sobre la Salud en Europa 2012 se centra de modo particular en el bienestar, cuestión que forma parte integral de la nueva estrategia sanitaria europea Salud 2020 - adoptada en septiembre de 2012 por los 53 estados miembros de la Región Europea de la OMS. El informe destaca que el bienestar y la salud son conceptos interactivos y multidimensionales, con algunos determinantes comunes, como por ejemplo los sistemas de salud. Garantizar la calidad de vida no corresponde a un solo sector o servicio, sino que un concepto multidimensional con múltiples determinantes. Se requiere un enfoque que implique la totalidad de los gobiernos y a la sociedad. La evidencia sugiere que la equidad en salud se consigue en la medida en que los gobiernos nacionales aceptan la responsabilidad de proteger y promocionar los derechos humanos, incluyendo por ejemplo, el cuidado sanitario, el saneamiento y la potabilización del agua, la protección social y la educación. Asimismo, una sociedad civil dinámica y comprometida puede mejorar la relevancia y la aceptación de las acciones, abordando la mejora de la equidad en salud (Blas et al., 2008).

Posteriormente, se han publicado dos nuevos Informes sobre la Salud en Europa (2015 y 2018). En el informe más reciente (Organización Mundial de la Salud, 2018), se muestra que el estado de la salud en Europa nunca ha estado mejor. Sin embargo, hay una realidad mejorable en las tendencias de los factores de riesgo para la salud y revela también una desigualdad persistente en la región y entre hombres y mujeres.

El sistema sanitario es también en sí mismo un determinante social de la salud, influenciado por e influenciando el efecto de otros determinantes sociales. El género, la educación, la ocupación, el nivel de ingresos, la etnicidad y el lugar de residencia están fuertemente relacionados con el acceso y beneficios del sistema sanitario. La Comisión de Determinantes de la Salud indica que la financiación de los sistemas sanitarios debe basarse en los impuestos generales, ya que la evidencia disponible se inclina a favor de la financiación pública como el mejor camino para reducir la inequidad. Por otro lado, los sistemas sanitarios muestran unos mejores resultados en salud cuando se basan en el desarrollo de la atención primaria y en una adecuada derivación a otros niveles de salud (Gwatkin et al., 2005), ya que están estratégicamente posicionados para evaluar los determinantes sociales de la salud y abordar a nivel comunitario la prevención primaria, lo que permitirá ahorrar costes, reducir la demanda y mejorar los resultados en salud (Allen et al., 2018).

Sucesos como la crisis económica del año 2008, tienen un impacto negativo tanto en los determinantes sociales de la población como en el propio sistema sanitario. Recientemente se ha publicado un análisis de las tendencias en los determinantes de la salud infantil (pobreza infantil, privación material) y los resultados perinatales (bajo peso al nacer y mortalidad infantil e inversión escolar en edades de 0 a 5 años) en países europeos entre los años 2005 y 2015, de acuerdo al nivel de austeridad impuesto por los gobiernos durante la época de crisis económica (Rajmil et al., 2018). La agrupación de los países fue la siguiente: $a$ ) nivel de austeridad alto: Dinamarca, 
Finlandia, Alemania, Noruega y Suecia; $b$ ) nivel de austeridad intermedio: Austria, Bélgica, Francia, Italia y Holanda, y c) nivel de austeridad bajo: Grecia, Islandia, Irlanda, Portugal, España y Reino Unido. En el Gráfico 1 se puede comprobar cómo aquellos países con mayor nivel de austeridad muestran peores resultados, sobre todo en el último periodo de estudio, tanto en los determinantes sociales como en los resultados en salud. Países donde se han implementado las medidas de austeridad más severas, han experimentado peores resultados en salud, como un mayor porcentaje de niños con bajo peso al nacer, y también ha aumentado la deprivación material, empeorando el impacto negativo que ya de por sí tiene la crisis económica. La conclusión del estudio es la urgente necesidad de proteger grupos vulnerables de la población como son los niños, del impacto de la austeridad.

\section{GRÁFICO 1}

TENDENCIAS EN LOS RESULTADOS PERINATALES (GRÁFICOS 1A Y 1B)

Y EN LOS DETERMINANTES DE LA SALUD INFANTIL (GRÁFICOS 1C, 1D

Y 1E) EN PAÍSES EUROPEOS DE ACUERDO AL NIVEL DE AUSTERIDAD IMPUESTO POR LOS GOBIERNOS DURANTE LA ÉPOCA DE CRISIS

ECONÓMICA (2005-2015)

GRÁFICO 1A

BAJO PESO AL NACER

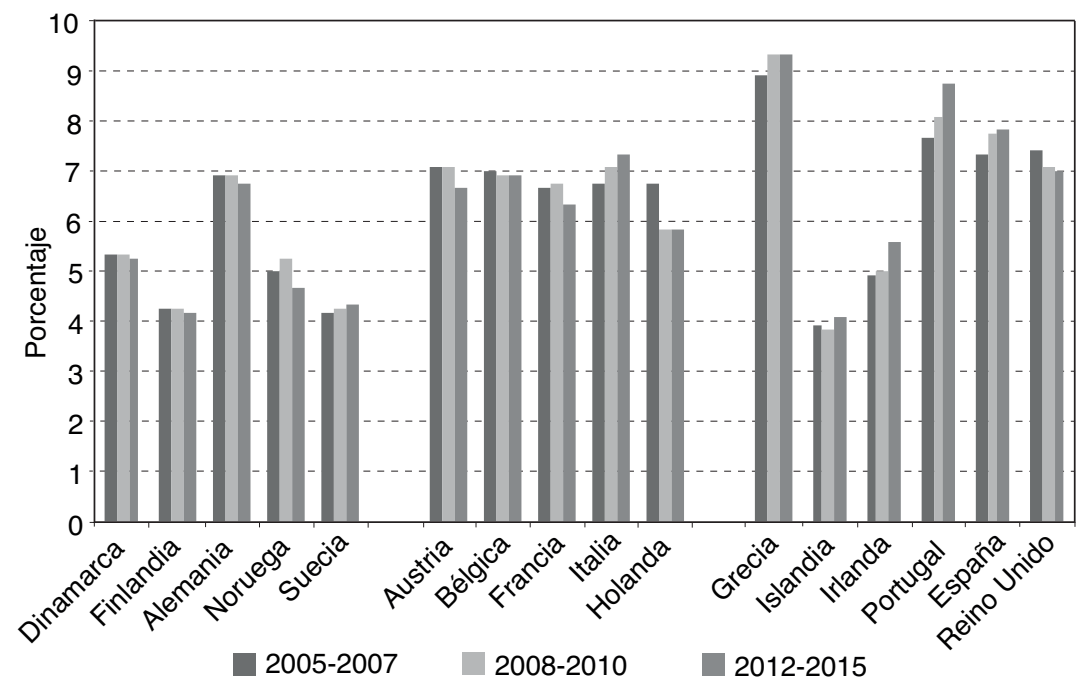

FUENTE: Elaboración propia a partir de datos de RAJMIL et al., 2018. 


\section{GRÁFICO 1B}

MORTALIDAD INFANTIL

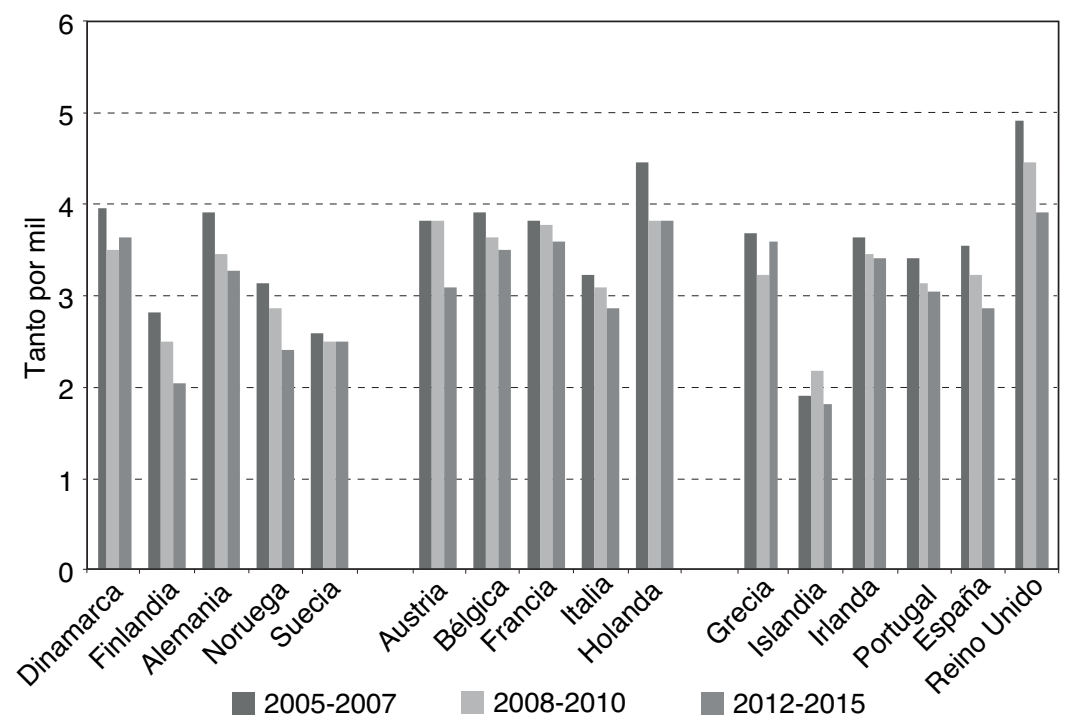

FUENTE: Elaboración propia a partir de datos de RAJMIL et al., 2018.

\section{GRÁFICO 1C}

POBREZA INFANTIL

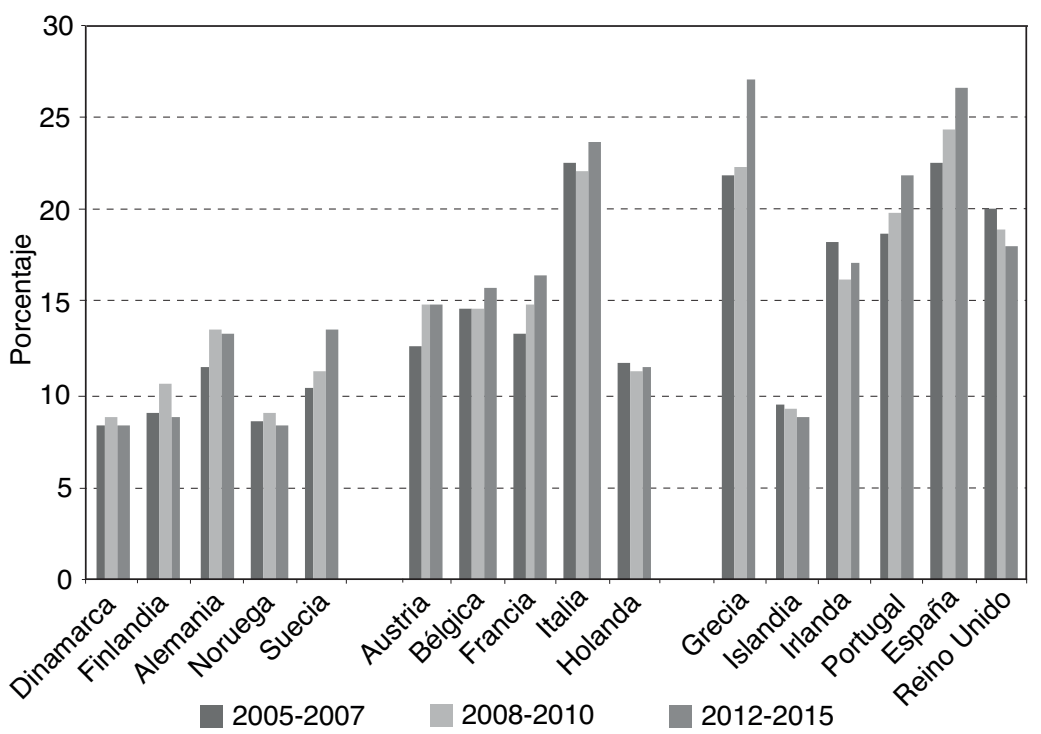

FUENTE: Elaboración propia a partir de datos de RAJMIL et al., 2018. 


\section{GRÁFICO 1D \\ DEPRIVACIÓN MATERIAL}

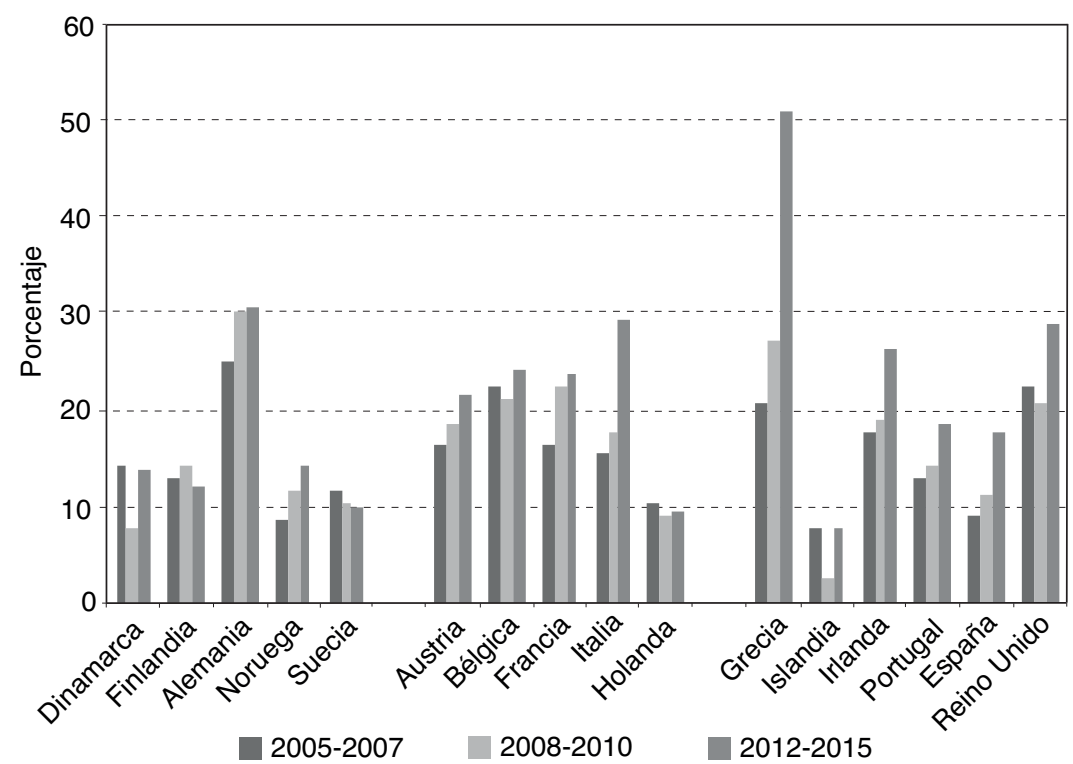

FUENTE: Elaboración propia a partir de datos de RAJMIL et al., 2018.

\section{GRÁFICO 1E}

\section{INVERSIÓN ESCOLAR}

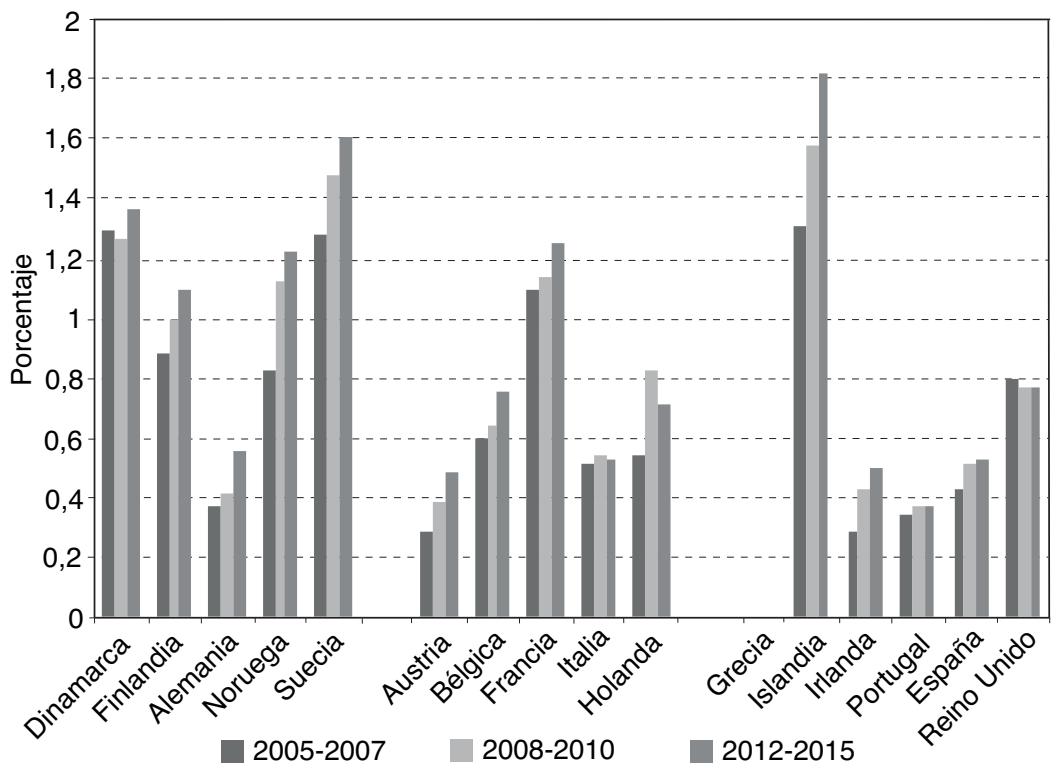

FUENTE: Elaboración propia a partir de datos de RAJMIL et al., 2018. 
NOTA: Resultados perinatales (datos de Organisation for Economic Co-operation and Development (OECD) Family Database (http://www.oecd.org/els/family/database.htm:

- Bajo peso al nacer: definido como el número de nacidos vivos pesando menos de 2500 gamos dividido por el número total de nacidos vivos.

- Mortalidad infantil: muertes de niños menores a 1 año por 1000 nacidos vivos.

Determinantes sociales de salud infantil:

- Pobreza infantil: porcentaje de niños viviendo en hogares con ingresos inferiores al 60 por 100de la mediana (datos de EU-Survey of Income and Living Conditions (EU-SILC) (http://ec.europa.eu/eurostat/web/microdataleuropean union-statistics-on-income-and-living-conditions).

- Tasa de deprivación material severa: proporción de niños menores de 18 años viviendo en familias con nivel primario de educación que no pueden permitirse pagar al menos cuatro de los nueve artículos considerados básicos, como tener atrasos en el pago de la hipoteca o del alquiler, facturas de servicios públicos, plazos de compra a plazos u otros pagos de préstamos; no poder pagar una semana de vacaciones anuales fuera de casa; no poder pagar una comida con carne, pollo, pescado (o equivalente vegetariano) cada dos días, etc. (datos de EU-Survey of Income and Living Conditions (EU-SILC)).

- Inversión escolar: porcentaje anual del producto interno bruto (PIB) de cada país (datos de OECD).

En resumen, y como objetivos futuros para mejorar la salud y el bienestar de la población es necesario reducir la inequidad en salud y asegurar el mantenimiento de sistemas de salud centrados en la persona.

\section{Género y salud}

La salud de las mujeres y los hombres es diferente: a pesar de que las mujeres tienen una mayor esperanza de vida que los hombres, viven menos años con buena salud (Krieger, 2003). También la salud es desigual entre hombres y mujeres, ya que existen determinantes sociales que establecen diferencias evitables (Borrel et al., 2004): la desigualdad según el género y según el nivel socioeconómico son dos aspectos que deberían evaluarse al mismo tiempo. La menor esperanza de vida de los hombres proviene tanto de diferencias biológicas en la susceptibilidad a la enfermedad como de distintos patrones de comportamiento en relación a los riesgos para la salud (Bird et al., 1999). La peor percepción que tienen las mujeres de su estado de salud refleja una mayor prevalencia de enfermedades crónicas, enfermedades físicas y mentales.

De acuerdo a los datos obtenidos en las Encuestas Nacionales de Salud que se han realizado en los últimos años en España (2001-2014), podemos observar las diferencias entre hombres y mujeres y según estado socioeconómico en la percepción que tiene la población acerca del estado de su salud. En el Gráfico 2 se analiza la diferencia entre el porcentaje de hombres y el de mujeres que valoran su estado de salud como positivo en los últimos 12 meses de acuerdo a su estado socioeconómico (la clase I es la clase más favorecida y la clase VI la más desfavorecida). Se puede comprobar como hay un mayor porcentaje de hombres que valoran su estado de salud como positivo que las mujeres (las diferencias en el porcentaje de las valoraciones entre hombres y mujeres son positivas). Aunque la tendencia en la diferencia entre 
hombres y mujeres en España ha disminuido en los últimos años, esta diferencia es todavía muy elevada en las clases sociales más desfavorecidas. Estas diferencias reflejan las relaciones de género.

Las mujeres españolas reciben, por lo general, una peor asistencia que los hombres, tanto en la etapa del diagnóstico como durante el tratamiento, sin olvidar que han sido tradicionalmente marginadas en los ensayos clínicos. Esto deriva de un planteamiento equivocado de igualdad o diferencia entre hombres y mujeres, lo que genera un comportamiento desigual en la atención sanitaria y/o en la investigación, conllevando a diagnósticos erróneos, sobre o infra prescripción de pruebas diagnósticas, fármacos, etc.

Evaluando también los datos de la Encuesta Nacional de Salud (años 2001-2014), se pueden observar las diferencias en el consumo referido de medicamentos en las últimas dos semanas entre mujeres y hombres según estado socioeconómico (Gráfico 3). Se puede comprobar cómo las mujeres refieren consumir más medicamentos que los hombres (las diferencias en el consumo referido entre mujeres y hombres

\section{GRÁFICO 2}

\section{DIFERENCIA ENTRE EL PORCENTAJE DE HOMBRES Y EL DE MUJERES QUE VALORAN SU ESTADO DE SALUD PERCIBIDO EN LOS ÚLTIMOS DOCE MESES COMO POSITIVO, SEGÚN CLASE SOCIAL (2001-2014)}

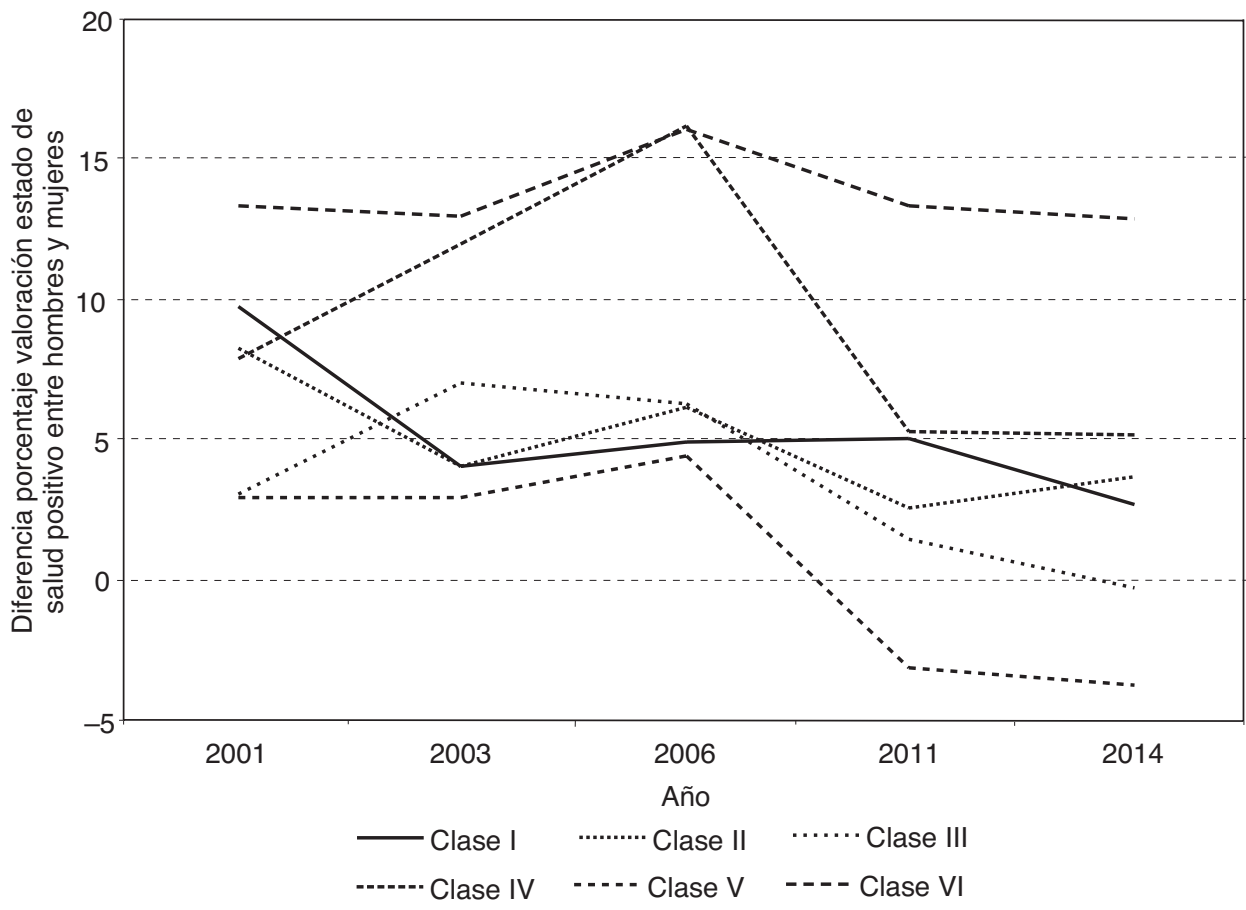

FUENTE: Elaboración propia a partir de datos de las Encuestas Nacionales de Salud, 2001-2014, Ministerio de Sanidad, Consumo y Bienestar Social.

NOTA: La Clase I es la más favorecida y la Clase VI la más desfavorecida. 


\section{GRÁFICO 3}

\section{DIFERENCIA EN EL CONSUMO DE MEDICAMENTOS REFERIDO EN LAS ÚLTIMAS 2 SEMANAS DE LAS MUJERES FRENTE A LOS HOMBRES SEGÚN CLASE SOCIAL (2001-2014)}

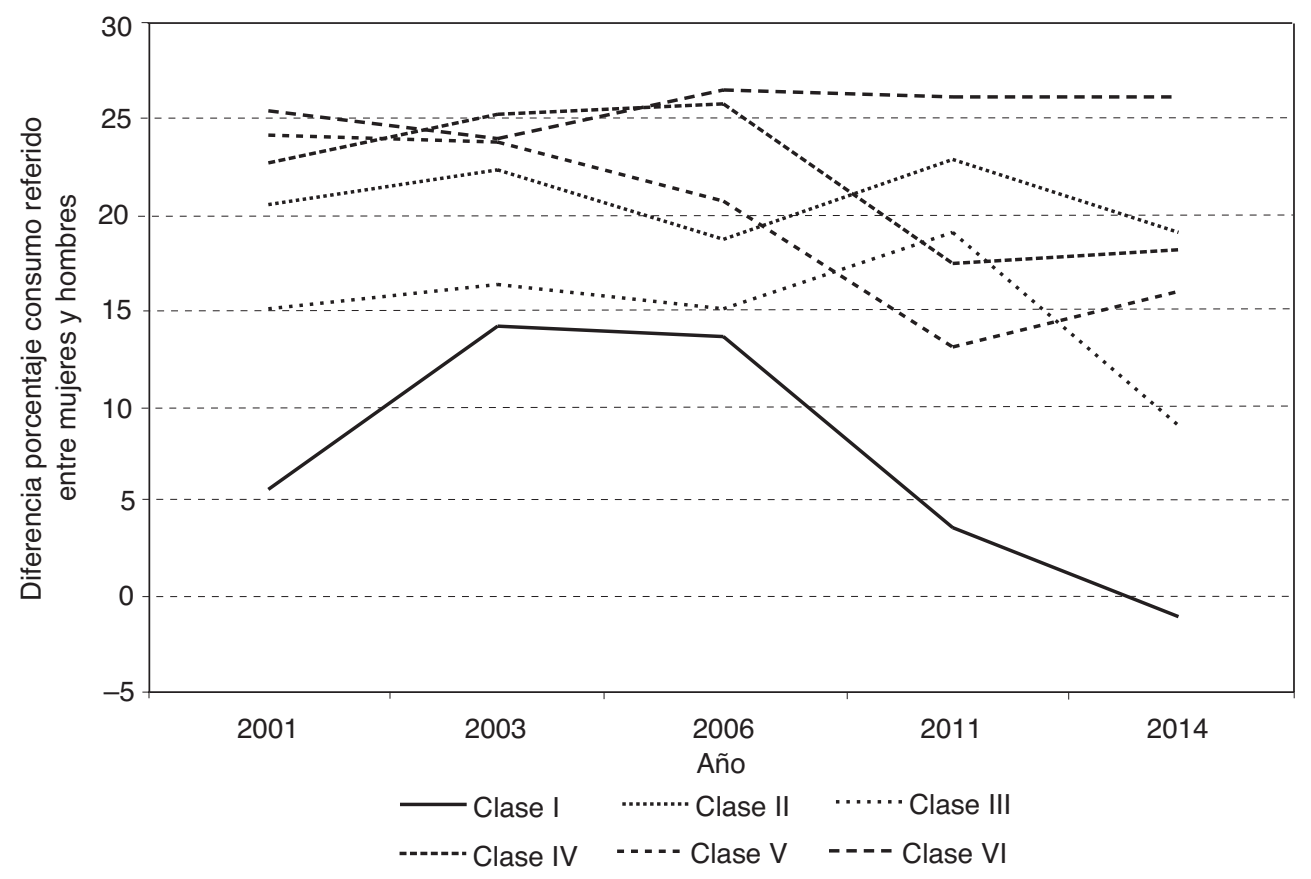

FUENTE: Elaboración propia a partir de datos de las Encuestas Nacionales de Salud, 2001-2014, Ministerio de Sanidad, Consumo y Bienestar Social.

NOTA: La Clase I es la más favorecida y la Clase VI la más desfavorecida.

son positivas). Las diferencias en el consumo referido entre mujeres y hombres en España han disminuido en los últimos años, excepto en las clases sociales más desfavorecidas, donde de acuerdo a los datos del año 2014 ha aumentado.

Por otro lado, hasta la fecha, no se ha puesto el suficiente énfasis en la interacción del género en la financiación en salud. La cobertura de salud universal enfatiza la equidad en los servicios; algunos grupos tienen mayores necesidades en salud y menor capacidad de financiación que otros. Esto implica que las necesidades de grupos vulnerables, como mujeres embarazadas, niños y enfermos crónicos, deberían recibir más atención que otros (Witter et al., 2017).

En resumen, las desigualdades por género en el ámbito de poder, recursos, normas y valores, y el modo de actuación de las organizaciones, daña la salud de millones de mujeres. El interés por la equidad en género está aumentando en la agenda de la Unión Europea y en la de sus países miembros, y las políticas para avanzar hacia una mayor igualdad están siendo implementadas en todos los niveles. No obstante, y aunque la posición de la mujer ha mejorado sustancialmente en las últimas déca- 
das en muchos países, todavía queda mucho por recorrer (Marmot et al., 2008). Por ejemplo, y de acuerdo a los resultados mostrados en el World Economic Forum's Global Gender Gap Report 2017, se estima que hasta dentro de 217 años no se cerrará la brecha del género en el lugar de trabajo a nivel global (World Economic Forum, 2017).

\section{Salud en Todas las Políticas}

La mejora en la salud de la población, no debe reducirse a mejoras del sistema de salud, sino que deben involucrarse todos los sectores de la sociedad. Salud en Todas las Políticas (STP) es un enfoque de la salud pública que, reconociendo que la salud puede verse influida por las decisiones adoptadas en sectores distintos del sanitario, evalúa de manera sistemática las implicaciones que tienen todas las decisiones políticas en la salud, busca sinergias y evita impactos negativos sobre la salud, con el objeto de mejorar la salud de la población y la equidad en salud (Conferencia de Helskinki, 2013).

El concepto de STP se basa en los principios de la promoción de la salud desarrollados inicialmente en la Declaración de Alma-Ata sobre Atención Primaria de Salud (1978) y en la Carta de Ottawa para la Promoción de la Salud (1986), desarrollados posteriormente en la Declaración de Adelaida del 2010 sobre Salud en Todas las Políticas. Estos principios fueron reforzados en la Declaración Política de Río sobre los Determinantes Sociales de la Salud del año 2011, la Declaración Política de la Reunión de alto nivel de las Naciones Unidas sobre las enfermedades no transmisibles del año 2011, y el Documento final aprobado en Rio+20 («El futuro que queremos») del año 2012. También están reflejados en muchos otros marcos, estrategias y resoluciones de la OMS, y contribuyen a la formulación de los objetivos de desarrollo post 2015.

Las razones para implementar la STP son numerosas. La primera de ellas es la evidencia de que se pueden obtener grandes beneficios en salud de políticas no sanitarias, dada la relación entre los determinantes sociales y la salud. La segunda razón se relaciona con la necesidad de una población sana como recurso esencial para el crecimiento y desarrollo socioeconómico. De esta manera, una política que tenga consecuencias negativas para la salud y el bienestar de la población impactará en la economía a través de la pérdida de productividad y del incremento de los costes del sistema sanitario. Las políticas exclusivas sobre los determinantes de la salud no son neutrales en términos de su efectividad en los distintos grupos sociales, beneficiándose menos los grupos desfavorecidos. Por eso, un enfoque en los determinantes de las desigualdades en salud asegura la mejora de la equidad en salud.

Algunos ejemplos de la implementación de esta estrategia a nivel global es el Proyecto de Ciudades Sostenibles, creado tras la Carta de Aalborg (1994) en la $1^{\mathrm{a}}$ Conferencia Europea de Ciudades y Pueblos Sostenibles, y los Compromisos de Aalborg en Río (2004), y que se ha visto relanzado tras la $8^{\text {a }}$ Conferencia Europea de 
Ciudades y Pueblos Sostenibles, celebrada en Bilbao en abril de 2016. A nivel europeo, la Unión Europea ha utilizado la implementación de la Evaluación del Impacto Sanitario (EIS) como una herramienta para identificar los vínculos entre la salud y otras políticas sectoriales. Destaca la Conferencia Mundial de Promoción de la Salud de Helsinki, del año 2013, con la declaración de Helsinki sobre Salud en Todas las Políticas. A nivel nacional, en Canadá y Australia se han dirigido a poblaciones específicas como los niños/as o a comunidades concretas (comunidades de bajos ingresos, escuelas, lugares de trabajo).

En España, el informe de la Sociedad Española de Salud Pública y Administración Sanitaria (SESPAS) de 2010 tuvo como objeto contribuir al avance de la incorporación del principio «Salud en todas las Políticas» en la salud pública. A pesar de estos avances, no hay en España una tradición asentada de políticas intersectoriales. Algún ejemplo positivo es la política de seguridad vial, donde las acciones intersectoriales han generado efectos beneficiosos para la salud o la Estrategia para la Nutrición, Actividad física y Prevención (NAOS). No obstante, el principal avance ha sido la aprobación de la Ley General de Salud Pública (Ley 33/2011, de 4 de octubre), en la que se establece que las administraciones públicas y los agentes privados estarán sujetos al principio de Salud en Todas las Políticas e introduce la Evaluación de Impacto en Salud en aquellas políticas que puedan afectar a la misma.

Otro ejemplo se encuentra en las políticas de mejora del medio ambiente. Así, se estimaron los beneficios para la salud y económicos de la mejora de la calidad del aire en 57 municipios del área metropolitana de Barcelona (Pérez et al., 2009). Los resultados del impacto en salud mostraron que los beneficios anuales de reducir la exposición media a PM10 $10^{1}$ se estimaban en 3.500 muertes menos (representando en media un aumento de la esperanza de vida de 14 meses), 1.800 ingresos hospitalarios menos por causas cardiorrespiratorias, 5.100 casos menos de bronquitis crónicas en adultos, 31.100 casos menos de bronquitis agudas en niños y 54.000 crisis asmáticas menos en niños y adultos. Los beneficios económicos totales se estimaron en una media de 6.400 millones de euros por año.

\section{Medicalización}

En los países desarrollados, y una vez que se ha garantizado el acceso a las necesidad básicas, ha aparecido una tendencia creciente a incluir dentro del ámbito sanitario situaciones que hasta la fecha eran ajenas al mismo. Un ejemplo ilustrativo se encuentra en la Asociación Americana de Psiquiatría, que tal y como denunció Lancet en el año 2012, incluía los sentimientos de duelo después de la muerte de un ser querido con duración superior a dos semanas como depresión y no como una reacción normal de duelo (Kleinman et al., 2012). Este proceso se ha denomina-

\footnotetext{
1 Partículas sólidas o líquidas de polvo, hollín, partículas metálicas o polen dispersas en la atmósfera y cuyo diámetro varía entre 2,5 y 10 micrómetros.
} 
do «promoción de enfermedades» (disease mongering en inglés), que se refiere al esfuerzo que realizarían las compañías farmacéuticas por llamar la atención sobre condiciones o enfermedades frecuentemente inofensivas con objeto de incrementar la venta de medicamentos mediante campañas publicitarias, visitadores médicos, estudios que intentan medicalizar cualquier dolencia, etc. (Moynihan et al., 2008).

Este hecho hace que el médico prescriba fármacos que el paciente no necesita, por lo que van a ser ineficaces cuando no perjudiciales, o solicite nuevas pruebas diagnósticas, originando nuevas consultas y nuevas intervenciones sanitarias, con el consiguiente aumento de coste y la posible presencia de efectos adversos. La Comisión Europea en su informe de 10 de diciembre de 2008 titulado Fortalecimiento de la farmacovigilancia para reducir los efectos adversos de los medicamentos, señala que en el conjunto de la Unión Europea, el 5 por 100 de los ingresos hospitalarios se deben a reacciones adversas a medicamentos, siendo la quinta causa de muerte en hospitales. Este mismo informe estima que en la UE, 197.000 muertes al año se deben a reacciones adversas a medicamentos (RAM) y que el coste total de estas RAM, para la sociedad, es de 79.000 millones de euros. Desde hace ya tiempo y desde distintos ámbitos de la medicina se está incidiendo en el control del uso excesivo de intervenciones clínicas por ejemplo, de las pruebas diagnósticas (Lumbreras et al., 2008). Choosing Wisely Australia ${ }^{\circledR}$ (una iniciativa que permite a los clínicos, consumidores e interesados en cuidado de la salud, iniciar conversaciones sobre exámenes, tratamientos y procedimientos innecesarios) y las Recomendaciones del National Institute for Health and Care Excellence, NICE, «do not do» (guías en las que además de lo que hay que hacer, se describe lo que hay que dejar de hacer o no hacer nunca), son solo dos de las muchas iniciativas que se han puesto en marcha para reducir este problema.

Siguiendo estas recomendaciones, en España y desde el año 2013, el Ministerio de Sanidad, junto con casi 50 sociedades científicas ha consensuado un catálogo de acciones clínicas que no deben llevarse a cabo, lo que supone un avance en el principio primum non nocere. Es desde luego un primer paso que va en consonancia con la evidencia que indica que por ejemplo, alrededor del 40 por 100 de las pruebas de imagen que se realizan son innecesarias (Vilar et al., 2018), si bien cabría que las administraciones sanitarias excluyesen de las prestaciones financiadas aquellas que no han demostrado beneficio alguno y aplicasen lo establecido en la Ley general de Salud Pública sobre la suspensión de acciones preventivas que no cumplan los objetivos para los que fueron diseñadas. Independientemente del abordaje desde la política sanitaria, también cabe plantearse cómo implantar este conocimiento en la práctica clínica. Sin embargo, todavía no se han diseñado las estrategias necesarias para conseguirlo (Berwick et al., 2017).

La responsabilidad de este hecho es compartida por la sociedad, los profesionales sanitarios, los medios de comunicación y la industria farmacéutica. En relación a la sociedad, se tiende a considerar que el sistema sanitario es el que debe garantizar los cuidados, perdiendo la capacidad del autocuidado y aumentando el consumo de recursos. Los medios de comunicación representan otra importante fuente de infor- 
mación sanitaria modulando las creencias y las expectativas de la sociedad. Se ha evidenciado la relación directa entre la aparición de una noticia médica en los medios y el uso de los servicios sanitarios por dicho motivo. También las instituciones políticas tienen la responsabilidad de gestionar la cartera de servicios e instaurar los instrumentos necesarios para limitar este tipo de efectos. Los profesionales sanitarios son los principales agentes de la medicalización, ya que tienen la capacidad de determinar qué diagnósticos se establecen y qué intervenciones se llevan a cabo. La sociedad, los medios de comunicación, etc., pueden fomentar la medicalización, pero son los profesionales sanitarios quienes finalmente la llevan a cabo. Por último, las industrias farmacéuticas tienen un papel principal en fomentar el uso, a través de la sociedad, los medios de comunicación, las instituciones políticas y los propios profesionales sanitarios, de una intervención farmacéutica o diagnóstica concreta. Cada vez que se genera una enfermedad, la consecuencia inmediata es que para cada proceso existe un tratamiento o un procedimiento diagnóstico nuevo.

La prevención cuaternaria previene la medicalización, disminuyendo la actividad médica innecesaria. Su objetivo es identificar pacientes con riesgo de sufrir procedimientos médicos excesivos, que conllevarían más daños que beneficios y ofrecerles alternativas aceptables (Albert et al., 2017). Para ello, entre las distintas intervenciones a llevar a cabo están aumentar el conocimiento de la población, de manera que puedan tomar decisiones sobre su propia salud al estar realmente informados, evitar confundir factores de riesgo con patologías, y suspender la difusión a través de los medios de comunicación u otros canales de intervenciones diagnósticas y terapéuticas de dudoso beneficio.

\section{Conclusiones/recomendaciones}

1. La mejora de la respuesta social a los problemas de salud debe ser abordada desde una perspectiva global, considerando que los problemas de salud más importantes tienen una dimensión global.

2. Para la universalización de la atención sanitaria es necesario implementar intervenciones que mejoren el sistema sanitario hasta alcanzar unos estándares de calidad basados en la evidencia disponible. Para ello, es esencial el desarrollo de la investigación operacional que evalúa la eficacia de las intervenciones en función de las características de cada sistema sanitario.

3. En el análisis de las prioridades en salud es fundamental una evaluación de los determinantes sociales, lo que conllevará a que las políticas de salud contribuyan a la reducción de las desigualdades sociales en salud.

4. El sistema sanitario es por sí mismo un determinante social que influye asimismo en otros determinantes. La reducción de la inequidad y la mejora de los resultados en salud pasa por la financiación pública del servicio sanitario y el desarrollo de la atención primaria.

5. La implementación del enfoque de Salud en Todas las Políticas contribuye 
a la mejora de la salud de la población a través del reconocimiento de que la salud puede verse influida por las decisiones adoptadas en sectores distintos al sanitario.

6. La introducción de la prevención cuaternaria en la práctica sanitaria es una acción de salud pública que contribuye a la disminución de la medicalización mejorando la salud de la población.

\section{Referencias bibliográficas}

[1] ALBER K.; KUEHLEIN T.; SCHEDLBAUER A. y SCHAFFER S. (2017). «Medical overuse and quaternary prevention in primary care-A qualitative study with general practitioners». BMC Fam Pract, 18, 99.

[2] ALLEN L. (2018). «Leveraging primary care to address social determinants». Health, 3, e466.

[3] ASAMBLEA GENERAL DE LAS NACIONES UNIDAS (Lancet Public) (2015). Resolución aprobada por la Asamblea General de las Naciones Unidas el 25 de septiembre de 2015; A/RES/70/1.

[4] BLAS E.; GILSON L.; KELLY M.P.; LABONTÉ R.; LAPITAN J.; MUNTANER C.; OSTLIN P.; POPAY J.; SADANA R.; SEN G.; SCHRECKER T. y VAGHRI,Z. (2008). «Addressing social determinants of health inequities: what can the state and civil society do?». Lancet, 372, 1684-9.

[5] BERWICK D. (2017). «Avoiding overuse-the next quality frontier». Lancet, 390, 102-104.

[6] BERWICK, D. M.; KELLEY E.; KRUK M. E.; NISHTAR, S. y PATE, M. A. (2018). «Three global health-care quality reports in 2018». Lancet, 392, 194-195.

[7] BIRD, C. E. y RIEKER, P. P. (1999). «Gender matters: an integrated model for understanding men's and women's health». Soc. Sci. Med., 48, 745-755.

[8] BORRELL, C.; GARCÍA-CALVENTE, M. M.; MARTÍ-BOSCÁ, J. V. y GRUPO DE GÉNERO Y SALUD PÚBLICA DE SESPAS (2004). «La salud pública desde la perspectiva de género y clase social». Gac. Sanit, 18, suppl. 1, 2-6.

[9] CLARK, J. (2014). «Medicalization of global health 1: has the global health agenda become too medicalized?». Glob. Health Action, 7, 23998.

[10] CONFERENCIA MUNDIAL DE PROMOCIÓN DE LA SALUD, HELSINKI, FINLANDIA (2013). La declaración de Helsinki sobre salud en todas las políticas. Recuperado en octubre 2018 de https://www.mscbs.gob.es/profesionales/saludPublica/pre Promocion/docs/DeclaracionHelsinki.pdf.

[11] CSDH, W. (2008). «Closing the gap in a generation: health equity through action on the social determinants of health». In: Final Report of the Commission on Social Determinants of Health. Geneva: WHO.

[12] GWATKIN, D. R.; WAGSTA, A. y YAZBECK, A. S. (eds.) (2005). Reaching the poor with health, nutrition, and population services. Washington, DC: World Bank.

[13] HERNÁNDEZ AGUADO, I. y LUMBRERAS, B. (2016). «Public-Private Partnerships: Role of Corporate Sponsorship in Public Health». En D. H. Barrett, L. H. Ortmann, A. Dawson, C. Sáenz, A. Reis y G. Bolan (eds.), Public Health Ethics: Cases Spanning the Globe. New York, Springer Open, 80-83. 
[14] KLEIMANN, A. (2012) «Culture, bereavement, and psychiatry». Lancet, 379, 608-9.

[15] KRIEGER, N. (2003). «Genders, sexes, and health: what are the connections-and why does it matter?». Int. J. Epidemiol., 32, 652-657.

[16] Ley 33/2011, de 4 de octubre, General de Salud Pública. BOE n. $.^{\circ} 240,5$ de octubre de 2011: 104593-626.

[17] LIM, S. S.; VOS, T.; FLAXMAN, A. D.; DANAEI, G.; SHIBUYA K. y ADAIR-ROHANI, H. et al. (2012). «A comparative risk assessment of burden of disease and injury attributable to 67 risk factors and risk factor clusters in 21 regions, 1990-2010: a systematic analysis for the Global Burden of Disease Study 2010». Lancet, 380, 2224-60.

[18] LUMBRERAS, B. y HERNÁNDEZ-AGUADO I. (2008). «El entusiasmo por las pruebas diagnósticas: efectos en la salud y formas de control». Gac. Sanit., 22, suppl. 1, 216-22.

[19] MARMOT, M.; FRIEL, S.; BELL, R.; HOUWELING, T. A.; TAYLOR, S. y COMMISION ON SOCIAL DETERMINANTS OF HEALTH. (2008). «Closing the gap in a generation: health equity through action on the social determinants of health». Lancet, 372, 1661-9.

[20] MOYNIHAN, R.; DORAN, E. y HENRY, D. (2008). «Disease Mongering Is Now Part of the Global Health Debate». PLoS Med, 5, 106.

[21] NACIONES UNIDAS (2015). «Objetivos de desarrollo del milenio informe de 2015. Recuperado en octubre 2018 de http://www.un.org/es/millenniumgoals/pdf/2015/mdgreport-2015_spanish.pdf.

[22] NACIONES UNIDAS (2017). «Objetivos de Desarrollo Sostenible». Recuperado en octubre 2018 de https:/repositorio.cepal.org/bitstream/handle/11362/40155/18/ S1700334_es.pdf.

[23] NUFFIELD COUNCIL ON BIOETHICS (2007). «Public health: ethical issues». London, Nuffield Council on Bioethics. Recuperado en noviembre 2018 de http://nuffieldbioethics.org/wp-content/uploads/2014/07/Public-health-ethical-issues.pdf.

[24] ORGANIZACIÓN MUNDIAL DE LA SALUD. OFICINA REGIONAL EUROPEA (2012). The European health report 2012: charting the way to well-being.

[25] ORGANIZACIÓN MUNDIAL DE LA SALUD. OFICINA REGIONAL EUROPEA (2018). «More than numbers-evidence for all». Recuperado en diciembre 2018 de $\mathrm{http}: / /$ www.euro.who.int/_data/assets/pdf_file/0008/379862/who-ehr-2018-eng.pdf?ua=1.

[26] OTTERSEN, O. P.; DASGUPTA J. y BLOUIN, C. et al. (2014). «The political origins of health inequity: prospects for change». Lancet, 383, 630-67.

[27] PÉREZ, L.; SUNYER, J. y KÜNZLI N. (2009). «Estimating the health and economic benefits associated with reducing air pollution in the Barcelona metropolitan area (Spain)». Gac Sanit., 23, 287-94.

[28] PETERS, D. H.; TRAN, N. T. y ADAM, T. (2013). Investigación sobre la implementación de políticas de salud: guía práctica. Alianza para la investigación en Políticas y Sistemas de Salud. Organización Mundial de la Salud.

[29] RAJMIL, L.; TAYLOR-ROBINSON, D.; GUNNLAUGSSON, G.; HJERN, A. y SPENCER, N. (2018). «Trends in social determinants of child health and perinatal outcomes in European countries 2005-2015 by level of austerity imposed by governments: a repeat cross-sectional analysis of routinely available data». BMJ Open, 8, e022932.

[30] ROWSON, M.; McCOY, D.; GUPTA, A. y DE NEGRI, A. (2004). «The Global Health Watch». PLoS Med, 1, e3. 
[31] THEOBALD, S.; BRANDES, N.; GYAPONG, M.; EL-SAHARTY, S.; PROCTOR, E.; DIAZ, T.; WANJI, S.; ELLOKER, S.; RAVEN, J.; ELSEY, H.; BHARAL, S.; PELLETIER, D. y PETERS, D. H. (2018). «Implementation research: new imperatives and opportunities in global health». Lancet, S0140-6736, 32205-0.

[32] VILAR-PALOP, J.; HERNÁNDEZ-AGUADO, I.; PASTOR-VALERO, M.; VILAR, J.; GONZÁLEZ-ÁLVAREZ, I. y LUMBRERAS, B. (2018) «Appropriate use of medical imaging in two Spanish public hospitals: a cross-sectional analysis». BMJ Open, 16, e019535.

[33] WORLD ECONOMIC FORUM (2017). «The global gender pay gap report 2017». Recuperado en octubre 2018 de https://www.weforum.org/reports/the-global-gender-gapreport-2017.

[34] WITTER, S.; GOVENDER, V.; RAVINDRAN, T. K. S. y YATES, R. (2017) «Minding the gaps: health financing, universal health coverage and gender». Health Policy Plan, 32, v4-v12. 